\title{
Haemodynamic and sound events preceding first heart sound in mitral stenosis ${ }^{1}$
}

\author{
J. B. Lakier, W. A. Pocock, G. E. Gale, and J. B. Barlow \\ From The Cardio-Vascular Research Unit, Department of Medicine, University of the \\ Witwatersrand, and The Cardiac Clinic, General Hospital, fohannesburg, South Africa
}

Phonocardiograms showed crescendo vibrations preceding $M_{1}$ in 23 patients with mitral stenosis and atrial fibrillation. In the 15 patients subjected to cardiac catheterization, simultaneous left atrial and left ventricular pressure tracings revealed that the vibrations occurred during the preisovolumic phase of left ventricular systole and that there was a synchronous rise in the left atrial pressure during this period.

An attempt is made to describe and explain the haemodynamic and sound events which precede the first major component $\left(M_{1}\right)$ of the first sound in mitral stenosis. Before the onset of left ventricular contraction, a mitral diastolic murmur results essentially from the pressure gradient from the left atrium to left ventricle. With sinus rhythm this gradient is increased by atrial systole and there is then an increase in both the velocity and the volume of flow through the stenosed valve orifice. With the onset of the preisovolumic phase of left ventricular systole, the pressure gradient decreases and it is abolished at the end of this period when the left ventricular and atrial pressures cross and the mitral valve orifice is 'closed'. Irrespective of whether the left atrium has contracted or is fibrillating, the crescendo vibrations which occur during the preisovolumic phase result essentially from a progressive diminution in the valve orifice size which in turn causes an increase in velocity, but decrease in volume, of flow until the point of valve closure.

It is well known that one of the most important factors influencing the intensity of a murmur is the velocity of blood flow across the site of its origin (McKusick, 1958a; Rodbard, 1964; Rushmer, 1970; Reddy, Shaver, and Leonard, 197I). It is thus understandable that accentuation of the mid-diastolic murmur in mitral stenosis occurs with atrial systole because this event increases the pressure gradient between the left atrium and the left ventricle. While the gradient is increased, both velocity and volume of flow through the stenosed valve will also be increased. The resulting murmur, which has been variously referred to as a 'presystolic' murmur (McKusick, 1958b), an 'atrial systolic' murmur (Raftery, 1965; Leatham, 1970), or as 'presystolicaccentuation' (Hurst and Cobbs, 1964), starts before the onset of left ventricular contraction and is crescendo to the first heart sound in patients with a relatively short PR interval and tight mitral stenosis, but may be crescendo-decrescendo where atrioventricular Received 4 April 1972.

1 This study was supported in part by The South African Medical Research Council. conduction is prolonged (Raftery, I965) or the degree of stenosis is mild.

Accentuation of a mid-diastolic murmur immediately preceding the first major component $\left(M_{1}\right)$ of the first heart sound has also been detected in mitral stenosis with atrial fibrillation. Explanations for this observation include postulates that the mid-diastolic murmur is interrupted by the first heart sound when diastole is short (Lewis, 1913; Ongley, Sprague, and Rappaport, 1955; Craige and Millward, 1971), that the murmur is produced by vibrations of the leaflets when the valve is pliable (Nichols et al., 1956), and that a tricuspid closure sound simulates accentuation of the murmur (Leo and Hultgren, 1959).

The purpose of this paper is to provide data which we believe indicate that the accentuation of a mid-diastolic murmur immediately before $M_{1}$ in mitral stenosis can be explained by an increased velocity, though decreased volume, of flow through the stenosed valve orifice during the interval between the onset of left ventricular contraction and the delayed closure of the mitral valve mechanism. This period is the preisovolumic phase of ven- 
tricular systole and is prolonged in mitral stenosis (Lakier et al., 1970).

\section{Subjects and methods}

Simultaneous left ventricular and left atrial pressure tracings, an external phonocardiogram, and standard lead II of the electrocardiogram, recor- ded during routine cardiac catheterization, were studied in 15 patients with tight mitral stenosis and atrial fibrillation. In 7 of the patients mild mitral incompetence was associated. Pressures were recorded on an Electronics for Medicine DR-I2 recorder through Statham $\mathrm{P}_{23} \mathrm{Db}$ strain gauge type transducers at paper speeds of 75 , 100 , and $150 \mathrm{~mm} / \mathrm{second}$ on sensitivities of 40

- and $100 \mathrm{mmHg}$. The external phonocardiogram, using an Electronics for Medicine Ps-I displacement microphone and a frequency response range of 40-2000 cycles/second, was recorded at the site of maximum intensity of $M_{1}$. The delay between the phonocardiographic and pressure recordings has previously been shown (Lakier et al., 1972a) to be less than $0.002 \mathrm{sec}$ and this can therefore

r be ignored.

In 8 other patients with tight mitral stenosis and atrial fibrillation, as well as in one of the 15 subjected to cardiac catheterization, phonocardiograms were recorded on a Cambridge 6-channel physiological recorder, type 72112 .

Phonocardiographic and cardiac catheterization observations In all 15 patients subjected to cardiac catheterization, vibrations which were crescendo from the time of onset of LV contraction were recorded on the Electronics for Medicine phonocardiograms. These crescendo vibrations started shortly after the $Q$ of the simultaneous electrocardiogram and coincided with a rise in left atrial pressure before the point of crossover (PCO) of the left atrial and left ventricular pressures (Fig. Ia). They were observed after relatively short diastolic pauses whereas after a long diastole, when there was equalization or near equalization of the left atrial and left ventricular pressures, no crescendo vibrations were recorded nor was there a rise of left atrial pressure (Fig. Ia). Similar vibrations, occurring after short diastolic pauses, were clearly shown in the 9 patients on whom phonocardiograms were recorded with the Cambridge apparatus (Fig. Ib).

$+$

\section{Discussion}

The mitral leaflets almost certainly appose at the time of PCO (Lakier et al., I972a) and we have previously produced evidence (Lakier et al., I972a, b) that after the mitral valve mechanism has 'closed' at PCO, the leaflets billow up into the left atrium causing the left atrial ' $c$ ' wave (LAC), at the peak of which tension on the leaflets and their chordae tendineae produces the major left-sided component $\left(M_{1}\right)$ of the first sound.

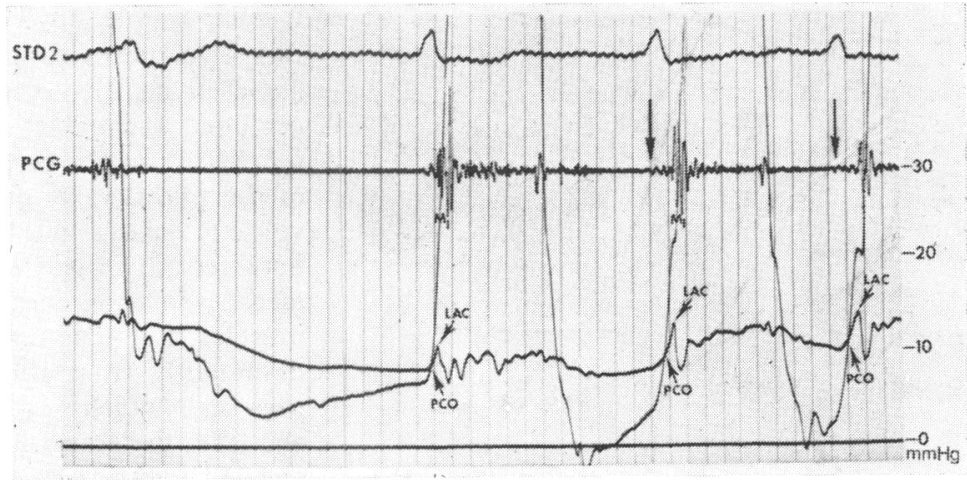

Ia
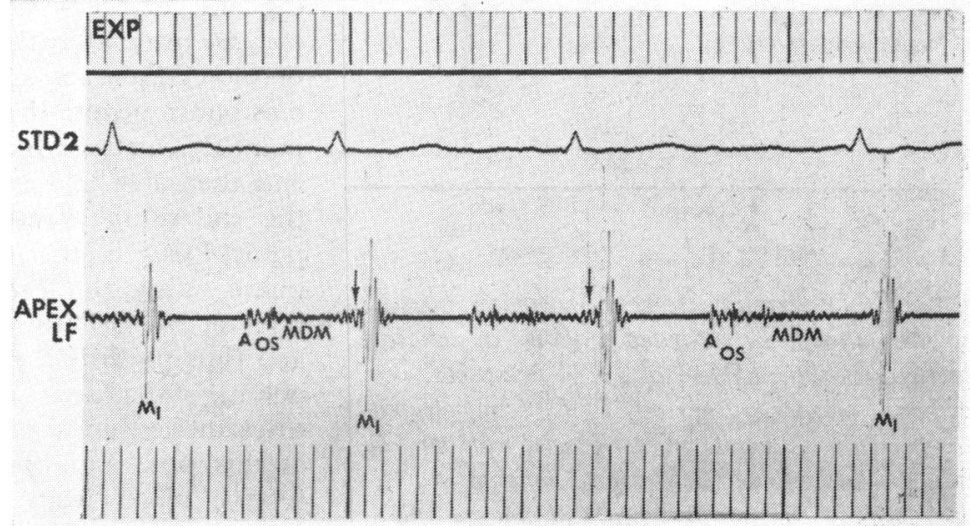

$\mathrm{Ib}$

FIG. I (a) Accentuation (long arrows) of a mid-diastolic murmur and a simultaneous rise in left atrial pressure are shown after the short diastolic pauses and precede the point (PCO) at which the left ventricular pressure exceeds that of the left atrium. After the long diastolic pause, no such vibrations are recorded and the left atrial pressure does not rise before PCO. LAC (the peak of the left atrial ' $c$ ' wave) coincides with the onset of $M_{1}$. (b) Crescendo vibrations (see arrows) of the mid-diastolic murmur (MDM) are shown on a low frequency (LF) Cambridge phonocardiographic tracing after the two relatively short diastolic pauses.

The changes in the functional anatomy of the mitral valve and left heart chambers which we believe precede $M_{1}$ in mitral stenosis with atrial fibrillation are shown diagrammatically in Fig. 2 in relation to the pressures and auscultatory features. After a short diastolic pause, and immediately before the onset (Fig. 2, point B) of left ventricular contraction, a pressure gradient is still present 


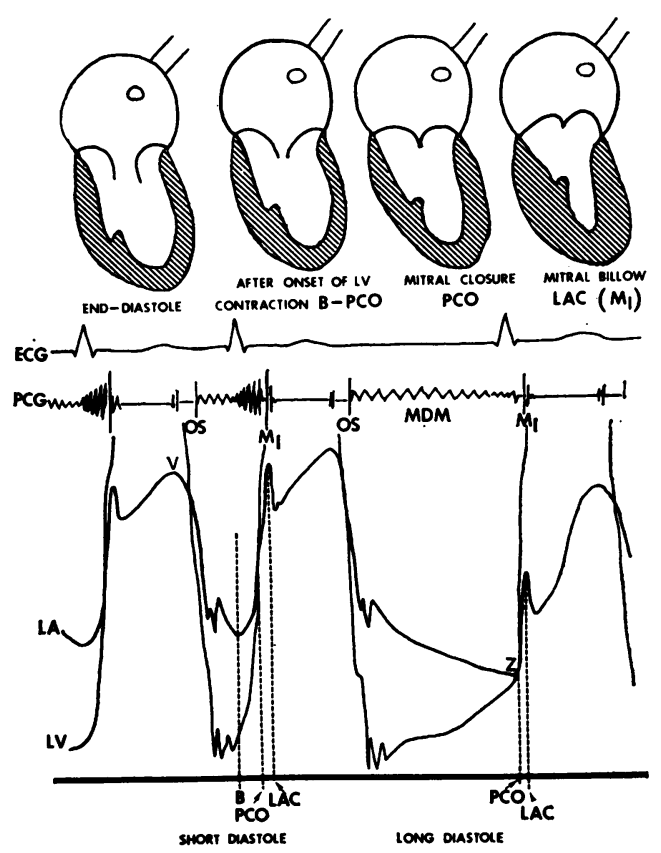

FIG. 2 Diagrammatic representation, based on simultaneously recorded tracings at cardiac catheterization, of $L A$ and $L V$ pressures, external phonocardiogram (PCG), and electrocardiogram of a patient with tight mitral stenosis and atrial fibrillation. Schematic diagrams of the two left heart chambers and the mitral leaflets correlate with the haemodynamic and sound events. Thus diagram 'end-diastole' represents the functional anatomical status after a short diastole while the stenosed mitral leaflets are 'wide open'; 'after onset of $L V$ contraction' illustrates the period $B-P C O$ during which the valve orifice progressively decreases because of the rising $L V$ pressure until it is occluded at 'mitral closure' (PCO). The rise in LA pressure and accentuation of the diastolic murmur are seen only after a short diastole. At 'mitral billow' the leaflets are further billowed up to produce LAC and the sound $M_{1}$. The period between valve closure (PCO) and $M_{1}$ is theoretically silent. For full description and explanation see text.

across the mitral valve but is decreasing. Though the stenosed mitral valve orifice is still fully 'open', the mid-diastolic murmur is becoming slightly softer because of the diminishing gradient and hence reduced velocity of flow through the valve. After the onset (Fig. 2, point B) of left ventricular contraction and throughout the preisovolumic phase (Fig. 2, interval B-PCO) of left ven- tricular systole, however, a change of the mitral valve mechanism supervenes in that the stenosed orifice decreases in size until it is occluded as the leaflets appose, and the valve is 'closed', at the point PCO. This reduction of the mitral valve orifice during the preisovolumic phase of left ventricular systole may depend in part on functional anatomical factors, such as contraction of the papillary muscles, but it almost certainly results predominantly from the rising left ventricular pressure which decreases and eventually abolishes the gradient between the left atrium and left ventricle at the point PCO. In spite of this pressure gradient decreasing throughout the preisovolumic phase, the diminishing valve orifice would result in a progressive increase in velocity, though decrease in volume, of blood flow across the valve. The mitral diastolic murmur thus accentuates until the point PCO, after which the orifice is occluded and the valve 'closed'. In addition to being the end of the preisovolumetric phase, the point PCO is the start of the isovolumic phase. While the leaflets are moving (Fig. 2, interval B-PCO) towards the closed position and thus towards the left atrial chamber, the volume of that cavity must decrease. However, since the left atrial pressure is relatively high at this time and the chamber continues to receive blood from the pulmonary venous system, the small rise in left atrial pressure during the preisovolumic contraction phase (Fig. Ia and Fig. 2, interval B-PCO) is understandable. During the period PCO-LAC $\left(\mathrm{M}_{1}\right)$, no murmur should be produced at the mitral valve (Fig. 2) but this time interval is extremely short in mitral stenosis (Lakier et al., 1972a) and the silent period is not detectable either clinically or phonocardiographically.

After a long diastolic pause these haemodynamic events are very different and are similar to those in atrial fibrillation without mitral stenosis. Since left atrial and left ventricular pressures now either approach equalization or have already equalized at the end of ventricular diastole (Fig. 2, point $Z$ ), the velocity of flow across the mitral valve is relatively low and the diastolic murmur is thus soft or absent (Fig. Ia and Fig. 2). After the onset of left ventricular contraction, PCO and valve closure take place almost immediately, the preisovolumetric phase of left ventricular systole is thus extremely short, no rise in left atrial pressure before PCO is seen, and no accentuation of the diastolic murmur is produced (Fig. Ia and Fig. 2).

Though in mitral stenosis with sinus rhythm it is the enhanced pressure gradient resulting from atrial systole which causes 
accentuation of the diastolic murmur before the onset of left ventricular contraction, when once the left ventricular pressure starts to rise the same alterations of the mitral valve * mechanism which take place with atrial fibrillation must again apply. The accentuation of an atrial systolic murmur thus continues throughout the left ventricular preisovolumetric contraction phase because of the progressive increase in velocity, though decrease in volume, of flow produced by the

- diminishing mitral orifice as the gradient between the left atrium and left ventricle is abolished.

There must be some mobility of the valve mechanism for a crescendo murmur of mitral stenosis to occur during the preisovolumic contraction phase, since the accentuation depends - essentially on a progressive decrease in size of the mitral valve orifice. In the event of a completely rigid mitral valve mechanism in which such decrease in size cannot take place, the velocity of blood flow would not increase when the left ventricular pressure rises to reduce and eventually to abolish both the left - atrial to left ventricular pressure gradient and the volume of flow through the fixed, stenosed valve orifice. Such a valve is unlikely to be completely competent, and since its rigid mechanism does not allow the leaflets to billow up into the left atrial cavity, the LAC is small and $M_{1}$ is relatively soft (Lakier et al., 1972a).

After this study was completed and originally submitted for publication, two important and relevant papers have come to our attention. Criley and co-workers have investigated the 'crescendo presystolic murmur' of I4 patients with mitral stenosis in sinus rhythm (Criley, Feldman, and Meredith, 197I) and of 4 patients in atrial fibrillation (Criley and Hermer, I97I). Using high speed cineangio-

Fraphy, these authors correlated observations of leaflet movement with simultaneously recorded left atrial and left ventricular pressures and external phonocardiograms. Though they do not specify the point in time at which the mitral valve closes and refer to 'closure' as an event which in mitral stenosis may last 0.05

- sec or longer, their hypothesis for the mechanism of production of the crescendo presystolic murmur is identical to ours. Criley and Hermer (I97I) have also shown and recognized the left atrial pressure rise during the crescendo phase of the murmur but do not discuss its mechanism. Finally, they emphasize that - 'rigid calcified valves, incapable of changes in orifice, would not be expected to generate a crescendo presystolic murmur in sinus rhythm or atrial fibrillation'.
We wish to thank Dr. H. M. Salmon, Superintendent of the Johannesburg General Hospital, for permission to publish.

\section{References}

Craige, E., and Millward, D. K. (1971). Diastolic and continuous murmurs. Progress in Cardiovascular Diseases, 14, 38.

Criley, J. M., Feldman, I. M., and Meredith, T. (197I). Mitral valve closure and the crescendo presystolic murmur. American fournal of Medicine, 51, 456.

Criley, J. M., and Hermer, A. J. (I97I). The crescendo presystolic murmur of mitral stenosis with atrial fibrillation. New England fournal of Medicine, 285, I284.

Hurst, J. W., and Cobbs, B. W. (1964). Diastolic rumbles. In The Theory and Practice of Auscultation, p. 396. Ed. by B. L. Segal. Blackwell Scientific Publications, Oxford.

Lakier, J. B., Fritz, V. U., Pocock, W. A., and Barlow, J. B. (I970). The left sided components of the first heart sound. South African Fournal of Medical Sciences, 35, 85 .

Lakier, J. B., Fritz, V. U., Pocock, W. A., and Barlow, J. B. (1972a). The mitral components of the first heart sound. British Heart fournal, 34, I60.

Lakier, J. B., Kinsley, R. H., Pocock, W. A., and Barlow, J. B. (1972b). Left atrial c wave and mitral leaflet size. Cardiovascular Research, 6, 585.

Leatham, A. (1970). Auscultation of the Heart and Phonocardiography, p. 138. J. and A. Churchill, London.

Leo, T., and Hultgren, H. (1959). Phonocardiographic characteristics of tight mitral stenosis. Medicine, 38, 85 .

Lewis, T. (1913). The time relations of heart sounds and murmurs, with special reference to the acoustic signs in mitral stenosis. Heart 4, 24I.

McKusick, V. A. (1958a). Cardiovascular Sound in Health and Disease, p. 132. Williams and Wilkens, Baltimore.

McKusick, V. A. (1958b). Cardiovascular Sound in Health and Disease, p. 295. Williams and Wilkens, Baltimore.

Nichols, H. T., Likoff, W., Goldberg, H., and Fuchs, M. (1956). The genesis of the 'presystolic' murmur in mitral stenosis. American Heart fournal, 52, 379.

Ongley, P. A., Sprague, H. B., and Rappaport, M. B. (1955). The diastolic murmur of mitral stenosis. New England fournal of Medicine, 253, 1049.

Raftery, E. B. (1965). The atrial systolic murmur in mitral stenosis. American fournal of Cardiology, 15, 735.

Reddy, P. S., Shaver, J. A., and Leonard, J. J. (r97I). Cardiac systolic murmurs: pathophysiology and differential diagnosis. Progress in Cardiovascular Diseases, 14, I.

Rodbard, S. (1964). The production and physical qualities of sound in the cardiovascular system. In The Theory and Practice of Auscultation, p. $33 \mathrm{Ed}$. by B. L. Segal. Blackwell Scientific Publications, Oxford.

Rushmer, R. F. (1970). Cardiovascular Dynamics, 3rd ed., p. 313. W. B. Saunders, Philadelphia.

Requests for reprints to Professor J. B. Barlow, Department of Medicine, General Hospital, Johannesburg, South Africa. 